\title{
Features of the chemokine profile of blood plasma by neurotoxic complications of acute lymphoblastic leukemia in children: preliminary report
}

\author{
Oksana Koryakina ${ }^{1 *}$, Vladimir Bazarnyi ${ }^{1}$, Larisa Fechina $^{2}$, Polina Kostromina $^{1}$, and Arina \\ Maksimova ${ }^{1}$ \\ ${ }^{1}$ Ural State Medical University, 620028 Repina str., 3, Yekaterinburg, Russia \\ ${ }^{2}$ Regional Children's Clinical Hospital, 620000, Yekaterinburg, Russian Federation
}

\begin{abstract}
The article presents an assessment of the neurotoxicity of chemotherapy in children with acute lymphoblastic leukemia receiving specific treatment according to the protocols used in pediatric oncological practice. An analysis of the neurological state with the determination of the chemokine profile of blood plasma was performed in 21 children aged 3 to 17 at the Regional Children's Clinical Hospital in Yekaterinburg and the Central Research Laboratory of the Ural State Medical University in 2019. In the study group of children, neurotoxic complications were recorded in $42.9 \%$ of cases. At the same time, the appearance of neurological symptoms in most patients $(77.7 \%)$ was observed during co chemotherapy at the stages of reinduction during consolidating treatment with a predominant clinical picture of chemo-induced polyneuropathy. In a comparative analysis of the indicators of the chemokine profile in groups of children, depending on the formation of neurotoxic complications during chemotherapy, we selected the chemokines CXCL10 (IP-10) and CXCL12 (SDF-1 $\alpha$ ) as possible prognostic biomarkers of damage to the nervous system.
\end{abstract}

\section{Introduction}

Hemoblastoses are one of the urgent problems of modern oncohematology. According to world statistics, the frequency of this pathology at children under the age of 15 years is 3.3 4.7 per 100 thousand child population. At the same time, there is a significant predominance of acute lymphoblastic leukemia up to $75-85 \%$. Modern methods for the treatment of hemoblastoses have improved the prognosis significantly, the 5-year survival rate presently is over $90 \%$ [1]. However, the use of chemotherapy is accompanied by a high frequency of drug complications, including those associated with neurotoxicity. The addition of neurological symptoms to the main clinical picture of the disease significantly aggravates the patient's condition, affects the prognosis and quality to life of the child. In

\footnotetext{
* Corresponding author: koryakina09@mail.ru
} 
recent years, this problem has been given special attention. Today, most research work is devoted to the analysis of the frequency, structure, and clinical features of chemo-induced neurological disorders [2-6]. The modern concept of the pathogenesis toxicity chemotherapeutic drugs is based on several types of effects on the nervous system: direct neurotoxicity, immune-mediated response and DNA damage [7-11]. Despite the active study of drug neurological complications, there has been a steady increase in the toxic effects of chemotherapy drugs. Now the issues of standardization of the management of such patients remain unresolved, the values of laboratory markers for predicting and early diagnosis of neurotoxic complications have not been determined, which defines the direction for further study of this problem.

\section{Purpose}

The aim of the study is assessing the nature of the damage to the nervous system from chemotherapy in children with acute lymphoblastic leukemia and to determine the specificity of chemokines in the blood for search to potential biomarkers of neurotoxicity as possible predictors of neurological complications. We analyzed the clinical picture and neurological status in 21 children aged 3 to 17 years with acute lymphoblastic leukemia (ALL), the children were treated at the Regional Center for Pediatric Oncology and Hematology in Yekaterinburg in 2019. The examination complex included a neurological examination, analysis of blood plasma with the determination of biomarkers that belong to the chemokine family.

\section{Materials and methods}

We analyzed the clinical picture and neurological status in 21 children aged 3 to 17 years with acute lymphoblastic leukemia (ALL), the children were treated at the Regional Children's Clinical Hospital in Yekaterinburg in 2019. The research complex included a neurological examination, analysis of blood plasma with the determination of biomarkers that belong to the chemokine family.

In the studied group of patients, the neurological status was assessed according to the generally accepted method. Neurological toxicity was assessed using the national Cancer Institute Common Terminology Criteria for Adverse Events (NCI-CTCAE) version 4.0 of 2010. All children were prescribed treatment according to program chemotherapy. The main category of patients received specific treatment according to the ALL-MB-2015 Protocol. Initial laboratory tests with chemokines were performed in all patients at different stages of chemotherapy $(\mathrm{n}=21)$ (study stage 1 ), and repeated tests were performed 1 month later in 11 patients (study stage 2). Taking into account the state of the nervous system during chemotherapy, two groups were formed among patients: group 1-patients with ALL who do not have neurological symptoms $(n=9)$; group 2 - patients with ALL who have neurotoxic complications $(\mathrm{n}=14)$.

Exclusion criteria: age of children under 3 years and older than 17 years, patients with a critical status for the main disease, patients with organic damage to the nervous system.

The following chemokine profile parameters were determined in all children: CXCL1 (GRO- $\alpha$ ), CXCL8 (interleukin-8, IL-8), CXCL10 (inducible protein-10, IP-10), CXCL12 (stromal cell factor $1 \alpha$, SDF-1 $\alpha$ ), CCL2 (monocytic chemotactic protein-1, MCP-1), CCL3 (macrophage inflammatory protein-1 $\alpha$, MIP-1 $\alpha$ ), CCL4 (macrophage inflammatory protein$1 \beta$, MIP-1 $\beta$ ) and CCL5 (chemokine expressed and secreted by T- cells upon activation RANTES). The concentration of chemokines was determined by multiparameter fluorescence analysis using magnetic microspheres (Xmap technology, Luminex) using 
Invitrogen test systems (eBioscience) and a Luminex 200 multiplex analyzer with xPONENT software.

Statistical processing of the material was carried out by the method of variation statistics using the Microsoft Excel program. The reliability of the results was evaluated using the non-parametric Mann-Whitney test. The differences were considered statistically significant at $\mathrm{p}<0.05$.

To assess the relationship between the laboratory parameters studied and the likelihood of developing neurological complications, the relative risk (RR) was calculated using a four-field table and an online calculator (website www.medcalc.org). Diagnostic characteristics - sensitivity and specificity - were determined using AtteStat 12.05, an addin to Microsoft Exel spreadsheets running the Microsoft Windows operating system.

\section{Results and discussions}

In the study group there were 21 children with a diagnosis of acute lymphoblastic leukemia (ALL), which was established on the basis of generally accepted methods. By gender, patients were distributed as follows: $52.4 \%$ of boys $(n=11), 47.6 \%$ of girls $(n=10)$. The category included children aged 3 to 17 years (average age was $7.6 \pm 4.3$ years). Among patients with ALL, the majority had a B-cell variant of the disease - 90.5\% ( $n=19)$. All children received chemotherapy according to the protocol.

It was found that symptoms of damage to the nervous system were observed in $42.9 \%$ of cases $(n=9)$, when we evaluated the neurological condition of the studied children Moreover, in all patients, neurological symptoms were recorded at the stages of chemotherapy, in most patients $77.7 \%(\mathrm{n}=7)$ during periods of reinduction of consolidation therapy, and in two people on the last days of the induction phase of treatment. 12 children $(57.1 \%)$ were in the group of patients who did not have disorders of the nervous system. The main contingent of this category of patients whose $83.3 \%(n=10)$ were at the stage of the first days of prescribed chemotherapy.

Among children with identified neurological disorders, patients with chemo-induced polyneuropathy predominated, $55.5 \%(\mathrm{n}=5)$, in three people $33.3 \%$ developed a clinic of acute cerebrovascular accident (ACA). It should be noted that in one patient a combination of neurological pathology was observed in the form of a combination of stroke with polyneuropathy. In one case, patients with symptoms of toxic encephalopathy and cephalgia were reported. When determining the severity of neurological disorders on the toxicity scale (NCI-CTCAE version 4.0 from 2010), it is seen that a weak, moderate and strong degree of neurotoxicity was detected with the same frequency (three people in each group).

In the analysis of patients with chemo-induced polyneuropathy, the clinical picture from the motor sphere was characterized by the appearance of subjective weakness or slight objective weakness in the lower extremities, without significant impact on functioning. In one case, there was a decrease in muscle strength up to 3 points in the proximal parts and up to 4 points in the distal parts of the lower extremities, which led to a violation of gait. All patients had suppressed Achilles tendon reflexes. Sensitive disorders were also registered in each child, and neuropathic pain prevailed, which was controlled by nonnarcotic drugs. In one patient, paresthesia was detected in the form of a "tingling" sensation in the distal parts of the upper extremities. According to the electroneuromyographic study, changes in peripheral nerves were detected in all the examined children. Signs characteristic of motor axonal neuropathy of the peroneal nerves were observed in three patients, the patient with the greatest motor deficit on the electroneuromiogram showed data for pronounced motor axonal-demyelinating polyneuropathy of the lower extremities. 
Indicators of electroneuromyography in two cases correspond to the violations, characteristic for sensory polyneuropathy of the lower extremities.

Acute cerebrovascular accident was the second most common neurotoxic complication in the study group. Two patients developed a clinic for sinus thrombosis of the Dura mater in the form of a General cerebral syndrome. One child developed an ischemic stroke in the basin of the left middle cerebral artery, which was accompanied by general cerebral symptoms and focal neurological symptoms corresponding to the area of brain damage. In two cases, ACA occurred during the induction stage of chemotherapy.

The development of chemo-induced lesions of the nervous system in children with acute lymphoblastic leukemia determines the need to study the mechanisms of their pathogenesis in order to develop new methods of diagnosis and treatment. In recent years, some attention has been paid to chemokines-factors that implement both inflammatory reactions and reparative capabilities of the nervous tissue. Studies have shown that chemokines play a significant role in the development of the nervous system, the induction and migration of neuronal progenitor cells, and the regulation of the blood-brain barrier [13,14]. In addition, there is evidence indicating the predictive value of the chemokine system in cancer patients. For example, these properties are set for CCL14 and CCL1, which are reliable predictive indicators. Other chemokines, such as CXCL1, CXCL8, and CXCL12, are adverse predictive markers [15]. Many processes of influence of chemokines on the state of nervous tissue when it is damaged are unknown, despite the existing attempts to decipher the understanding of the complex system of chemokines in functioning in relation to the nervous system. Many processes of influence of chemokines on the state of nervous tissue when it is damaged are unknown, despite the existing attempts to decipher the understanding of the complex system of chemokines in functioning in relation to the nervous system.

We evaluated the chemokine profile in children with ALL. 21 people were examined at the initial stage of chemotherapy (study phase 1), a month later, a second study was conducted with an assessment of chemokine indices in 11 children (study phase 2). A comparative analysis of the mean values of chemokines in the studied groups showed that no significant differences were found in all parameters (table 1). It can be noted that the course of the disease and chemotherapy have no effect on the state of the chemokine profile during the period of dynamic observation, taking into account the data obtained. It is possible that such results are related to the peculiarities of pathogenetic mechanisms of the studied pathology or to the shortness of the stage of comparative analysis of chemokine indicators, which was 1 month. In general, order for obtain obvious conclusions about the dependence of the chemokine status on the oncological process and the administration of chemotherapy drugs, it is necessary to increase the study population in groups with examinations during further catamnestic observation.

Table 1. The concentration of chemokines in the blood of children with ALL at different stages of therapy, $\mathrm{M} \pm \mathrm{m}$.

\begin{tabular}{|l|c|c|}
\hline Index $\times$ pg/ml & Research stage $1(\mathrm{n}=21)$ & Research phase 2 $(\mathrm{n}=11)$ \\
\hline CXCL1(GRO- $\alpha)$ & $99,72 \pm 39,14$ & $87,32 \pm 11,70$ \\
\hline CXCL8 (IL-8) & $47,42 \pm 12,15$ & $80,52 \pm 24,84$ \\
\hline CXCL10 (IP-10) & $265,92 \pm 55,68$ & $202,35 \pm 32,89$ \\
\hline CXCL12 (SDF-1 $\alpha)$ & $1647,51 \pm 99,43$ & $1583,56 \pm 136,53$ \\
\hline CCL2 (MCP-1) & $503,20 \pm 76,70$ & $411,27 \pm 143,50$ \\
\hline CCL3 (MIP-1 $\alpha)$ & $20,97 \pm 4,76$ & $21,10 \pm 1,81$ \\
\hline CCL4 (MIP-1 $\beta)$ & $50,08 \pm 13,88$ & $75,96 \pm 8,67$ \\
\hline CCL5 (RANTES) & $694,19 \pm 237,85$ & $619,58 \pm 81,27$ \\
\hline
\end{tabular}

$* \mathrm{p}<0,05$. 
The next aspect was to compare the level of chemokines in groups of patients with the development of neurotoxic complications during chemotherapy (table 2). The most noticeable change was in the form of a 2 -fold increase in level $(\mathrm{p}<0.05)$ of CXCL10 (IP-10) in the group of patients with neurological disorders was observed when comparing these indicators in children who don't have changes in the nervous system (group 1) with patients who have formed neurological disorders (group 2). Also in this group, a slight increase in CXCL12 (SDF-1AA) by $10 \%(\mathrm{p}<0.05)$ was detected. Therefore we can distinguish the index CXCL10 (IP-10), which may have a certain diagnostic value in the group of children with chemo-induced neurological disorders.

Table 2. Comparative analysis of the level of chemokines in children with ALL depending on the development of chemo-induced neurological disorders, $\mathrm{M} \pm \mathrm{m}$.

\begin{tabular}{|l|c|c|}
\hline \multicolumn{1}{|c|}{ Groups } & $\begin{array}{l}\text { Group 1 (n=9) } \\
\text { patients without neurological } \\
\text { disorders }\end{array}$ & $\begin{array}{l}\text { Group 2 (n=14) } \\
\text { patients with neurological } \\
\text { disorders }\end{array}$ \\
\hline CXCL1(GRO- $\alpha$ ) & $175,80 \pm 85,26$ & $70,96 \pm 8,48$ \\
\hline CXCL8 (IL-8) & $79,58 \pm 31,34$ & $39,55 \pm 9,96$ \\
\hline CXCL10 (IP-10) & $142,01 \pm 29,12$ & $288,86 \pm 47,91^{*}$ \\
\hline CXCL12 (SDF-1 $\alpha)$ & $1562,75 \pm 166,43$ & $1721,41 \pm 103,35^{*}$ \\
\hline CCL2 (MCP-1) & $474,92 \pm 170,91$ & $16,50 \pm 88,91$ \\
\hline CCL3 (MIP-1 $\alpha)$ & $16,91 \pm 2,07$ & $59,56 \pm 2,09$ \\
\hline CCL4 (MIP-1 $\beta$ ) & $56,30 \pm 12,97$ & $518,31 \pm 6,08$ \\
\hline CCL5 (RANTES) & $517,68 \pm 79,06$ & \\
\hline
\end{tabular}

* When comparing indicators in groups 1 and $2 \mathrm{p}<0,05$.

For reason that that chemokines, which level significantly differed in the groups of patients with neurotoxic complications, may have different diagnostic value, indicators of relative risk, sensitivity and specificity of these parameters were calculated (table 3 ). At the same time, it was noted that the indicators CXCL10 (IP-10) and CXCL12 (SDF-1) had a fairly high level of relative risk, which indicates their significant association with the development of neurological disorders.

Table 3. Diagnostic characteristics of the level of certain chemokines in neurotoxic complications in children with ALL.

\begin{tabular}{|l|c|c|c|c|}
\hline Index & Critical value & $\begin{array}{c}\text { Relative risk } \\
\text { (RR) }\end{array}$ & Sensitivity & Specificity \\
\hline $\begin{array}{l}\text { CXCL10 } \\
\text { (IP-10) }\end{array}$ & $>140 \mathrm{pkg} / \mathrm{ml}$ & 2,292 & 71,4 & 77,4 \\
\hline $\begin{array}{l}\text { CXCL12 } \\
\text { (SDF-1 } \alpha)\end{array}$ & $>1200 \mathrm{pkg} / \mathrm{ml}$ & 3,200 & 75,0 & 66,7 \\
\hline $\begin{array}{l}\text { CXCL10 } \\
+\end{array}$ & $>140 \mathrm{pkg} / \mathrm{ml}$ & 2,821 & 78,6 & 77,8 \\
CXCL12 & $>1200 \mathrm{pkg} / \mathrm{ml}$ & & & \\
\hline
\end{tabular}

Wherein, the sensitivity of the CXCL10 test (IP-10) and the specificity of CXCL12 (SDF-1 $\alpha$ ) were not very high. At the same time, the simultaneous use of two parameters provided significant sensitivity $(78.6 \%)$ and specificity $(77.8 \%)$ for the diagnosis of neurological disorders in children with ALL. The results on changes in the level of these chemokines in chemo-induced neurological complications are of particular significance, taking into account the known information about their participation not only in the processes of formation of nerve tissue and regulation of the blood-brain barrier, but also in the mechanisms of neuroinflammation $[14,16]$. 


\section{Findings}

Our research shows that in children with acute lymphoblastic leukemia receiving chemotherapy, almost half of the cases (42.9\%) form neurotoxic complications, which mainly develop at the stages of reinduction of consolidating therapy in $77.7 \%$ of patients. The predominance of chemo-induced lower limb polyneuropathy among all neurological complications was observed in $55.5 \%$ of patients. The study of the chemokine profile in children with acute lymphoblastic leukemia revealed stability indicators against the background of the course of the disease and chemotherapy during the follow-up period, which amounted to 1 month. Probably, such results are associated with the complexity of the pathogenetic mechanisms of the studied pathology; for convincing conclusions, it is necessary to replenish patients in groups with the study of chemokines during periods of prolonged dynamic observation. In general, considering the issue of chemo-induced neurotoxicity in acute lymphoblastic leukemia in children, given the small cohort of patients, it is difficult to determine the pathogenetic and diagnostic significance of chemokines. However, noting the non-specificity of chemokine reactions, we identified chemokines CXCL10 (IP-10) and CXCL12 (SDF-1 $\alpha$ ) as potential predictors / markers of neural tissue damage. The combined use of these two parameters had high sensitivity (78.6\%) and specificity $(77.8 \%)$ for the diagnosis of neurotoxicity at chemotherapy drugs. Thus, the results of a study on the state of the chemokine profile in children with ALL determine the direction for further study this problem in order to establish the diagnostic value of chemokines as laboratory predictors for predicting and early detection of chemoinduced neurological complications.

\section{References}

1. A.G. Rumyantsev, Ped. Hem/Onc. Immunopath., 14, 5-15 (2015)

2. E. A. Politova, N. N. Zavadenko, A. G. Rumyantsev, Oncohem., 4, $24-31$ (2013)

3. M. G. Sharipova, D. S.Smirnov, M. R. Yakupov, I. I. Spichak, Ped. Bull. S. Ur., 1, 53-61 (2016)

4. E. I. Kuznetsova, Path. Phys. Exp. Ther., 58, 71-77 (2014)

5. S. Guram, E. Richards, B. Messahel, Arch. Dis. Child., 101 (2016)

6. E.V. Vilchevskaya, Onc., 4, 212-214 (2002)

7. T.A. Ahles, A.J. Saykin, Nat. Rev. Cancer, 7, 192-201 (2007)

8. K.W. Kelley, R.M. Bluthe, R. Dantzer, J.H. Zhou, W.H. Shen, R.W. Johnson, S.R. Broussard, Brain. Behav. Immun., 17, 112-118 (2003)

9. M. Yang, J.S. Kim, J. Kim, H. Wang, T. Shin, C. Moon, Biochem. Pharmacol., 82, 72-80 (2011)

10. F. Yan, J.J. Liu, V. Ip, S.M.F. Jamieson, M.J. McKeage, J. Neurochem., 135, 1099$1112(2015)$

11. H.S. Kim, C. Guo, E.L. Thompson, Y. Jiang, M.R. Kelley, M.R. Vasko, S.H. Lee, Mutat. Res. (2015)

12. A. Calls, V. Carozzi, X. Navarro, L. Monza, J. Bruna, Exp. Neurol., 68,145-155 (2019)

13. J.L. Williams, D.W. Holman, R.S. Klein, Front. Cel.1 Neurosci., 8, 1-12 (2014)

14. A.E.S. Watson, K. Goodkey, T. Footz, A. Voronova, Neurosci. Lett., 715 (2020)

15. H.T.T. Do, C.H. Lee, J. Cho, Cancers, 12, 2 (2020)

16. Y. Zhou, H. B. Cao, W. J. Li, L. Zhao, Chin. J. Nat. Med., 16, 11 (2018) 